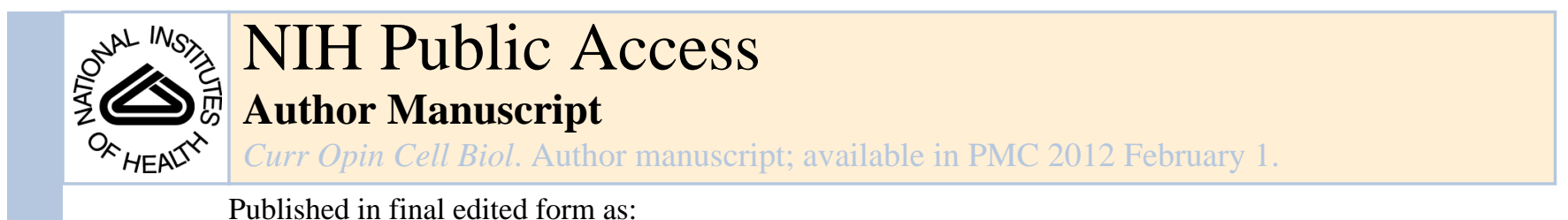

Curr Opin Cell Biol. 2011 February ; 23(1): 109-113. doi:10.1016/j.ceb.2010.08.007.

\title{
Mechanisms of Aneuploidy
}

\author{
Duane A. Compton \\ Department of Biochemistry, Dartmouth Medical School, Hanover, NH 03755; Norris Cotton \\ Cancer Center, Lebanon, NH 03766, USA
}

\section{INTRODUCTION}

Genome integrity relies on the equal partitioning of replicated chromosomes to daughter cells during cell division. Errors in chromosome segregation lead to aneuploidy, a state where the number of chromosomes in a cell or organism deviates from multiples of the haploid genome. Aneuploidy arising through chromosome mis-segregation during meiosis is a major cause of infertility and inherited birth defects. Approximately one third of miscarriages are aneuploid and it is estimated that $10-30 \%$ of all human fertilized eggs are aneuploid (1). The frequency of karyotypic problems increases dramatically during mitotic divisions in early embryos where $\sim 80 \%$ of human embryos possess aneuploid blastomeres $\left(2^{*}\right)$. In brain tissue, approximately one third of mouse neuroblasts are aneuploid (3) and there is an elevated rate of aneuploidy in neuronal and non-neuronal cells in human brains relative to lymphocytes (4). Interestingly, the aneuploid neurons appear to be functional (5). Moreover, approximately $90 \%$ of solid tumors are aneuploid (6), and it has been speculated since the $19^{\text {th }}$ century that aneuploidy in somatic tissues is a mechanism driving tumorigenesis (6-9). These high rates of aneuploidy in normal and abnormal tissue underscore the importance of determining the mechanisms causing chromosome missegregation. Moreover, given that chromosome mis-segregation is essentially irreversible, it is critical to understand how cells respond to aneuploidy and how tissue context contributes to the fate of aneuploid cells.

\section{Mechanisms of chromosome mis-segregation}

Chromosome segregation is a mechanical process that relies on the structural integrity of the microtubule spindle apparatus and a checkpoint signaling pathway to ensure high fidelity. The organization of spindle microtubules into bipolar arrays with focused poles is important for the accuracy of chromosome segregation. Chromosomes can't segregate if microtubules are organized into a monopolar configuration and cell division with multipolar spindles will

(C) 2010 Elsevier Ltd. All rights reserved.

Department of Biochemistry, 413 Remsen Bldg., Dartmouth Medical School, Hanover, N.H. 03755, Tel: (603) 650-1990, FAX: (603) 650-1128, duane.a.compton@dartmouth.edu.

Publisher's Disclaimer: This is a PDF file of an unedited manuscript that has been accepted for publication. As a service to our customers we are providing this early version of the manuscript. The manuscript will undergo copyediting, typesetting, and review of the resulting proof before it is published in its final citable form. Please note that during the production process errors may be discovered which could affect the content, and all legal disclaimers that apply to the journal pertain.

Preview

Accurate chromosome segregation during cell division is essential for genome integrity. Errors in chromosome segregation are irreversible and lead to a state of aneuploidy where the number of chromosomes in a cell or organism is not a multiple of the haploid number of chromosomes. Aneuploidy reduces fecundity and is a frequent cause of inherited birth defects. In addition, aneuploidy is very common in solid tumors where it is associated with poor patient prognosis. Recent work has revealed the most common pathways by which chromosomes mis-segregate leading to aneuploidy. Moreover, answers to the key question of how cells respond to aneuploidy are beginning to emerge. 
partition chromosomes to more than two daughter cells, a condition that has been shown to have lethal consequences for all the daughter cells $\left(10^{*}\right)$. The structural integrity of the microtubule attachment sites on chromosomes is also critical to segregation fidelity. Spindle microtubules attach to chromosomes through specialized structures called kinetochores that assemble on each sister chromatid adjacent to the centromere. In human cells, each kinetochore binds approximately 25 microtubules. The separation of sister kinetochores by centromeric chromatin ensures their back-to-back geometry, and this geometric constraint ensures segregation accuracy by promoting attachment of each kinetochore to microtubules oriented toward only one spindle pole. The timing of sister chromatid separation at anaphase is regulated by a checkpoint signaling pathway that monitors the attachment of spindle microtubules to kinetochores. This checkpoint prevents sister chromatid separation until all kinetochores have bound sufficient numbers of microtubules. Finally, cytokinesis divides the cell into two daughters, each with a normal complement of chromosomes. Failure in any of these processes increases the probability of chromosome mis-segregation leading to aneuploidy.

Chromosome segregation is the most conspicuous event in the cell cycle and insights into the mechanisms causing chromosome mis-segregation have come from the direct examination of chromosome movement using live cell imaging techniques. FISH analyses show that the mis-segregation rate of chromosomes in aneuploid tumor cells is 20- to 100fold higher than non-transformed diploid cells $(11,12 * *)$. This high level of mis-segregation is called chromosomal instability (CIN) and the persistent mis-segregation of chromosomes at high rates probably causes aneuploidy in tumors with karyotypes in the range of 40-60 chromosomes (13). Live cell imaging showed that the most common cause of chromosome mis-segregation in aneuploid tumor cells with CIN is lagging chromosomes at anaphase $\left(12^{* *}\right)$ and not defects in the spindle assembly checkpoint as had been previously reported (14). Kinetochores on the lagging chromosomes have microtubules oriented toward both spindle poles in an arrangement called merotely (15). Interestingly, fixed cell analyses of mouse neuroblasts also show higher levels of lagging chromosomes in anaphase (3) suggesting that aneuploidy in neuronal tissue may also be driven by persistent merotelic attachment of kinetochores.

Merotelic kinetochore-microtubule (kMT) attachments arise spontaneously during early phases of mitosis as a consequence of the stochastic nature of kMT interactions (16;Figure 1A). Moreover, since merotelic kinetochores achieve appropriate numbers of microtubule attachments, they effectively satisfy the spindle assembly checkpoint and do not prevent anaphase onset (15). Thus, it is incumbent on cells to correct merotelic kMT attachments prior to anaphase onset to ensure faithful chromosome segregation. The overall prevalence of merotelic kinetochores is driven by two rates - the rate of their formation and of their correction - and aneuploid tumor cells have been shown to have defects in both rates (13). For example, many tumor cells have extra centrosomes (17). Centrosomes participate in spindle pole organization and extra centrosomes induce the transient formation of multipolar spindles in early mitosis. That disruption of spindle morphology greatly exaggerates the number of kinetochores that form microtubule attachments to multiple poles (Figure 1B). Prior to anaphase onset, the centrosomes coalesce to form bipolar spindles (18), but the transient multipolar state vastly increased the number of merotelic attachments and increases chromosome mis-segregation rates $(10 *)$. The correction of merotelic kMT attachments relies on the release of improperly attached microtubules and direct analyses have shown that kMT attachments in aneuploid tumor cells are excessively stable, compromising their ability to correct erroneous kMT attachments (19;Figure 1C). Indeed, faithful chromosome segregation can be restored to otherwise unstable aneuploid cells by increasing the correction capacity of kinetochores by overexpression of enzymes that specifically destabilize kMT attachments $\left(20^{* *}\right)$. This result demonstrates a direct causal relationship 
between defects in $\mathrm{kMT}$ attachments and chromosome mis-segregation that drives tumor cell aneuploidy.

Live cell imaging has also recently been used to directly examine chromosome missegregation mechanisms in mammalian oocytes $(21 * *)$. The incidence of aneuploidy increases dramatically as women age indicating that there is an age-associated deterioration in oocyte quality (1). It was recently shown that sister chromatid cohesion at centromeres is compromised in meiosis I oocytes from old mice but not in oocytes from young mice. The loss of cohesion correlated with reduced quantities of the cohesion subunit Rec8 associated with chromosomes, and fits with data from other model systems showing that reduced cohesion can increase mis-segregation rates in oocytes $(22,23)$. The reduction of centromeric cohesion disrupts the normal geometric constraint that favors the attachment of kinetochores to spindle microtubules from opposing spindle poles. Thus, oocytes from older mice have a greatly enhanced frequency with which the kinetochores attach to spindle microtubules oriented toward the wrong spindle pole and this elevates chromosome mis-segregation rates. A similar pathway has been recently documented in somatic cells that are lacking the function of the Retinoblastoma $(\mathrm{Rb})$ tumor suppressor gene $\left(24^{*}, 25^{*}\right)$. Loss of Rb function compromises centromere cohesion by reducing the quantity of the condensin subunit CAPD3 associated with chromosomes. This attenuates the back-to-back geometric constraint on sister kinetochores and greatly increases the frequency of merotelic attachments that elevates chromosome mis-segregation rates leading to aneuploidy (24*;Figure 1D). This may be how mutant forms of cohesin proteins cause CIN (26).

Finally, some aneuploid tumor cells have karyotypes that are near the tetraploid number of chromosomes. In these cases, it is likely that the cells arrived at an aneuploid karyotype by progressing through a transient tetraploid state (9), and two mechanisms can generate tetraploidy. First, cell-to-cell fusion results in a tetraploid hybrid (27). Viruses can drive cell fusion and this mechanism has been demonstrated to induce aneuploid cancer cells (28). Second, failure of cytokinesis generates tetraploid cells (29). Tetraploid cells in these contexts are only transient and are probably not chromosomally stable due to excessively high rates of merotelic kinetochore formation induced by the presence of extra centrosomes.

In summary, various events contribute to chromosome mis-segregation that leads to aneuploidy including defects in spindle geometry, kMT dynamics, chromosome cohesion, and cytokinesis. Interestingly, despite differences in their molecular underpinnings, most of these mechanisms converge on a common pathway to cause chromosome mis-segregation by undermining the appropriate attachment of kinetochores to spindle microtubules. Perhaps it should not be surprising that defects in kMT attachments are the most common mechanism causing chromosome mis-segregation since erroneous merotelic kMT attachments evade detection by the spindle assembly checkpoint.

\section{Consequences of aneuploidy}

The chromosome segregation machinery is designed for high fidelity to maintain the euploid content of the cell or organism. Chromosome mis-segregation rates differ dramatically between cell types and organisms. For example, single cell organisms are quite fastidious with budding yeast mis-segregating a chromosome only once every million cell divisions. In contrast, human somatic cells mis-segregate a chromosome approximately every 100 cell divisions $\left(12^{* *}, 30\right)$. Even more extreme, human eggs are notoriously sloppy with rates of aneuploidy estimated at $20 \%$, and the incidence increases dramatically with maternal age (1). These rates demonstrate that, in some cell types, aneuploidy arises at substantial frequencies and recent work has shown that the cellular response to aneuploidy varies depending on the organism and the cellular context. 
In a recent study haploid budding yeast were engineered to carry an extra copy of a single chromosome $\left(31^{* *}\right)$. Disomic strains remain viable indicating that aneuploidy, per se, is not lethal in this organism. However, the growth rate of the strains carrying an extra chromosome was retarded relative to the haploid strain and the growth retardation was independent of the identity of the disomic chromosome. The genes on the disomic chromosome were expressed and there was an energetic burden on the aneuploid strains to synthesize and then degrade superfluous proteins encoded on the extra chromosome. Thus, disomy in an otherwise haploid strain did not reduce viability, but it generated a metabolic burden that reduced fitness of the cells compared to their normal counterparts. As an organism that grows as single cells it is easy to envision how strains with reduced fitness would be easily outcompeted by surrounding euploid cells in the population. These authors have also shown that trisomic fibroblasts derived from mouse embryos carrying Robertsonian translocations also grow slower than diploid cells (32). The effect of this growth reduction on the development and/or fitness of multicellular organisms remain unknown, but it was argued that growth retardation is a generalized response to trisomy that would lead to poor fitness of the aneuploid individuals such as has been described in other model organisms such as plants and fruit flies (33).

At the other end of the spectrum, approximately $20 \%$ of all human eggs are aneuploid, yet a sizeable proportion of those eggs remain competent for fertilization resulting in aneuploid embryos. Thus, eggs have mechanisms to cope with aneuploidy to maintain viability and escape any potential growth retardation. Perhaps the large maternally loaded stores of all components to support egg function offsets the expression of superfluous proteins from aneusomic chromosomes. Nevertheless, most aneuploid mammalian embryos do not develop properly and it is well documented that monosomies and most trisomies fail embryonic development (1). This represents the major cause for reduced fecundity in humans, and demonstrates that when aneuploidy arises at the time of fertilization that it severely compromises embryonic development. In contrast, a recent study has shown that approximately $80 \%$ of early human embryos obtained through in vitro fertilization contain aneuploid blastomeres despite the fact that most $(87.5 \%)$ single cell fertilized oocytes were euploid under the same conditions $(2 *)$. These data suggest that chromosome segregation during the early mitotic divisions is particularly error prone which may reflect the relaxation of checkpoint activity to accommodate rapid embryonic cell divisions. Strikingly, the take home rate of healthy babies following in vitro fertilization is much higher than would be predicted based on such high frequencies of aneuploidy. This indicates that there is selection against the aneuploid cells later during development.

Finally, somatic mammalian cells do not readily propagate following chromosome missegregation suggesting that otherwise diploid cells are intolerant of aneuploidy $\left(12^{* *}, 34^{*}\right)$. The intolerance of aneuploidy helps maintain the karyotype of the population despite a rather high mis-segregation rate $\left(\sim 1: 100 ; 12^{* *}, 34^{*}\right)$. However, it is well established that a majority of solid tumors are aneuploid, and recent analyses have shown a high frequency of aneuploid neuroblasts and neurons $(3,4)$. Indeed, aneuploid neurons appear to contribute to neural signaling networks indicating that they are fully functional (5). Thus, somatic cells in the context of a tumor or a developing nervous system have developed tolerance for aneuploidy. The mechanisms promoting tolerance of aneuploid neural cells are not known. In contrast, recent work with cultured somatic cells has shown that loss of the p53 tumor suppressor pathway is one mechanism to promote aneuploid cell growth $(34 *)$. That work showed that both chromosome loss or gain activates p53 to suppress growth of cells that mis-segregate chromosomes. The p53 protein is probably stabilized through a pathway that involves activation of $\mathrm{p} 38$ stress kinase and it suppresses cell cycle progression by activation of the p21 cyclin-dependent kinase inhibitor. 
In summary, our understanding for how euploid cells respond to chromosome missegregation remains limited and this is a fertile area that deserves attention. Given that cellular context plays an important role in how cells cope with an aneuploid genome, there are probably multiple pathways involved that need to be identified and explored.

\section{Conclusions and Future Perspectives}

In conclusion, recent work has illuminated the most common mechanisms that cause chromosome mis-segregation in somatic cells and in mammalian oocytes. A common theme emerging is that many of them involve defects in the attachment of spindle microtubules to kinetochores. This emphasizes the importance of identifying the molecular events that regulate the attachment dynamics of kinetochore-bound microtubules. Finally, it is important to identify the mechanisms that provide cells with tolerance to aneuploidy and to determine how those mechanisms are influenced by tissue context. That knowledge could open new therapeutic strategies to selectively target aneuploid tumor cells.

\section{Acknowledgments}

I thank Sarah Thompson for comments on the manuscript and her contribution of the figure. Work in the author's lab is supported by the National Institutes of Health (GM51542).

\section{References}

1. Hassold T, Hunt P. To err (meiotically) is human: the genesis of human aneuploidy. Nat Rev Genet 2001;2:280-291. [PubMed: 11283700]

2. Vanneste E, Boet T, Le Caignec C, Ampe M, Konings P, Melotte C, Debrock S, Amyere M, Vikkula M, Schuit F, Fryns J-P, Verbeke G, K'Hooghe R, Moreau Y, Vermeesch JR. Chromosome instability is common in human cleavage-stage embryos. Nat Med 2009;15:577-583. [PubMed: 19396175]. This work demonstrated high rates of chromosome mis-segregation in early embryos.

3. Rehen SK, McConnell MJ, Daushal D, Kingsbury MA, Yang AH, Chun J. Chromosomal variation in neurons of the developing and adult mammalian nervous system. Proc Natl Acad Sci USA 2001;98:133361-133366.

4. Rehen SK, Yung YC, McCreight MP, Kaushal D, Yang AH, Almeida BSV, Kingsbury MA, Cabral DMS, McConnell MJ, Anliker B, Fontanoz M, Chun J. Constitutional aneuploidy in the normal human brain. J Neurosci 2005;25:2176-2180. [PubMed: 15745943]

5. Kingsbury MA, Friedman B, McConnell MJ, Rehen SK, Yang AH, Kaushal D, Chun J. Aneuploid neurons are functionally active and integrated into brain circuitry. Proc Natl Acad Sci USA 2005;102:6143-6147. [PubMed: 15837924]

6. Weaver BAA, Cleveland DW. Does aneuploidy cause cancer? Curr. Opin. Cell Biol 2006;18:658667. (2006). [PubMed: 17046232]

7. von Hansemann D. Ueber asymmetrische zelltheilung in epithelkrebsen und deren biologische bedeutung. Virschows Arch. Pathol. Anat 1890;119:299-326.

8. Wolf, U. Theodor Boveri, and his book on the problem of the origin of malignant tumors. In: German, J., editor. Chromosomes and Cancer. New Your: Wiley; 1974. p. 3-20.

9. Ganem NJ, Storchova Z, Pellman D. Tetraploidy, aneuploidy and cancer. Curr Opin Genet Dev 2007;17:157-162. [PubMed: 17324569]

10. Ganem NJ, Godinho SA, Pellman D. A mechanism linking extra centrosomes to chromosomal instability. Nature 2009;460:278-282. [PubMed: 19506557]. The authors show that multiple centrosomes increase the incidence of merotelic attachments by causing transient multipolar spindles and that anaphase with multipolar spindles is lethal to all the daughter cells.

11. Lengauer C, Kinzler KW, Vogelstein B. Genetic instability in colorectal cancers. Nature 1997;386:623-627. [PubMed: 9121588]

12. Thompson SL, Compton DA. Examining the link between chromosomal instability and aneuploidy in human cells. J Cell Biol 2008;180:665-672. [PubMed: 18283116] . Used time-lapse microscopy 
to show that the most common cause of chromosomal instability in human tumor cells is persistent merotelic attachment of microtubules to kinetochores.

13. Thompson SL, Bakhoum SF, Compton DA. Mechanisms of chromosomal instability. Curr Biol 2010;20:R285-R295. [PubMed: 20334839]

14. Cahill DP, Lengauer C, Yu J, Riggins GJ, Willson JK, Markowitz SD, Kinzler KW, Vogelstein B. Mutations of mitotic checkpoint genes in human cancers. Nature 1998;392:300-303. [PubMed: 9521327]

15. Cimini D, Howell B, Maddox P, Khodjakov A, Degrassi F, Salmon ED. Merotelic kinetochore orientation is a major mechanism of aneuploidy in mitotic mammalian tissue cells. J Cell Biol 2001;153:517-527. [PubMed: 11331303]

16. Cimini D, Moree B, Canman JC, Salmon ED. Merotelic kinetochore orientation occurs frequently during early mitosis in mammalian tissue cells and error correction is achieved by two different mechanisms. J Cell Sci 2003;116:4213-4225. [PubMed: 12953065]

17. Nigg EA. Centromsome aberrations: cause or consequence of cancer progression? Nat Rev Cancer 2002;2:815-825. [PubMed: 12415252]

18. Quintyne NJ, Reing JE, Hoffelder Dr, Gollin SM, Saunders WS. Spindle multipolarity is prevented by centrosomal clustering. Science 2005;307:127-129. [PubMed: 15637283]

19. Bakhoum SF, Genovese F, Compton DA. Deviant kinetochore microtubule dynamics underlie chromosomal instability. Curr Biol 2009;19:1937-1942. [PubMed: 19879145]

20. Bakhoum SF, Thompson SL, Manning AL, Compton DA. Genome stability is ensured by temporal control of kinetochore-microtubule dynamics. Nat Cell Biol 2009;11:27-35. [PubMed:

19060894] . The authors demonstrated that high fidelity chromosome segregation can be restored to tumor cells with CIN by expression of enzymes that destabilize kinetochore-microtubule attachments.

21. Chiang T, Duncan FE, Schindler K, Schultz RM, Lampson MA. Evidence that weakened centromere cohesion is a leading cause of age-related aneuploidy in oocytes. Curr Biol. In press. . The authors used time-lapse imaging of oocytes from old and young mice to show that the most common cause of age-associated nondisjunction was the deterioration of centromere cohesion which led to improper attachments of spindle microtubules to kinetochores.

22. Hodges CA, Revenkova E, Jessberger R, Hassold TJ, Hunt PA. SMC1beta-deficient female mice provide evidence that cohesins are a missing link in age-related nondisjunction. Nat Genet 2005;37:1351-1355. [PubMed: 16258540]

23. Subramanian VV, Bickel SE. Aging predisposes oocytes to meiotic nondisjunction when the cohesin subunit SMC1 is reduced. PLoS Genet 2008;4:e1000263. [PubMed: 19008956]

24. Manning AL, Longworth MS, Dyson NJ. Loss of pRB causes centromere dysfunction and chromosomal instability. Genes Dev 2010;24:1364-1376. [PubMed: 20551165] . Provides evidence that the RB tumor suppressor protein regulates centromere cohesion to promote accurate chromosome segregation.

25. Coschi CH, Martens AL, Ritchie K, Francis SM, Cakrabarti S, Berube NG, Dick F. Mitotic chromosome condensation mediated by the retinoblastoma protein is tumor-suppressive. Genes Dev 2010;24:1351-1363. [PubMed: 20551166] . Provides evidence that the RB tumor suppressor protein regulates centromere cohesion to promote accurate chromosome segregation.

26. Barber, t; McManus, K.; Yuen, KWY.; Reis, M.; Parmigiani, G.; Shen, D.; Barrett, I.; Nouhi, Y.; Spencer, F.; Markowitz, S.; Velculescu, VE.; Kinzler, KW.; Vogelstein, B.; Lengauer, C.; Hieter, P. Chromatid cohesion defects may underlie chromosome instability in human colorectal cancers. Proc Natl Acad Sci USA 2008;105:3443-3448. [PubMed: 18299561]

27. Duelli D, Lazebnik Y. Cell-to-cell fusion as a link between viruses and cancer. Nat Rev Cancer 2007;7:968-976. [PubMed: 18034186]

28. Duelli D, Padilla-Nash HM, Berman D, Murphy KM, Ried T, Lazebnik Y. A virus causes cancer by inducing massive chromosomal instability through cell fusion. Curr Biol 2007;17:431-437. [PubMed: 17320392]

29. Fujiwara T, Bandi M, Nitta M, Ivanova EV, Bronson RT, Pellman D. Cytokinesis failure generating tetraploids promotes tumorigenesis in p53-null cells. Nature 2005;437:1043-1046. [PubMed: 16222300] 
30. Cimini D, Tanzarella C, Degrassi F. Differences in malsegregation rates obtained by scoring anatelophases or binucleate cells. Mutagenesis 1999;14:563-568. [PubMed: 10567031]

31. Torres EM, Sokolosky T, Tucker CM, Chan LY, Boselli M, Dunham MJ, Amon A. Effects of aneuploidy on cellular physiology and cell division in haploid yeast. Science 2007;317:916-924. [PubMed: 17702937]. The authors developed yeast strains carrying single extra chromosomes and observed that those aneuploid strains suffered growth deficiencies. This result was independent of the identity of the aneusomic chromosome.

32. Williams BR, Prabhu VR, Hunter KE, Glazier CM, Whittaker CA, Housman DE, Amon A. Aneuploidy affects proliferation and spontaneous immortalization in mammalian cells. Science 2008;322:703-709. [PubMed: 18974345]

33. Torres EM, Williams BR, Amon A. Aneuploidy: cells losing their balance. Genetics 2008;179:737-746. [PubMed: 18558649]

34. Thompson SL, Compton DA. Proliferation of aneuploid human cells is limited by a p53-dependent mechanism. J Cell Biol 2010;188:369-381. [PubMed: 20123995] . The authors used somatic cells to show that diploid cells activate a p53 response to suppress proliferation following missegregation of a single chromosome. 


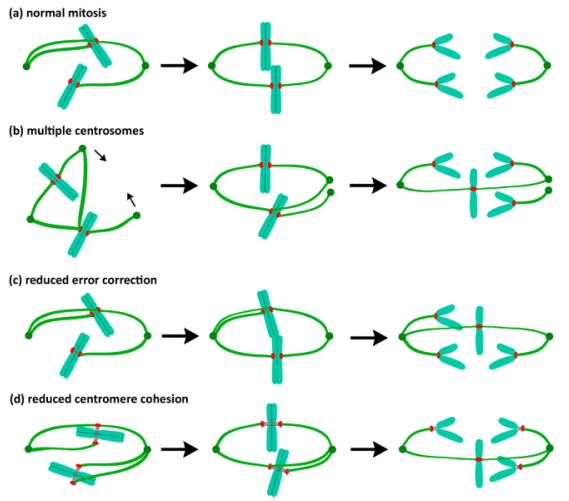

Figure 1.

Mechanisms for chromosome mis-segregation during cell division. Many mechanisms causing chromosome mis-segregation arise from defects in the attachments of spindle microtubules to kinetochores. (a) Errors in kinetochore-microtubule attachments occur spontaneously in early phases of mitosis but are efficiently corrected in normal cells to promote accurate chromosome segregation. (b) Extra centrosomes increase the frequency of kinetochore-microtubule attachment errors by inducing transient defects in spindle geometry. (c) Correction of erroneous kinetochore-microtubule attachments relies on release of improperly attached microtubules and hyperstable kinetochore-microtubule attachments reduces the efficiency of correction. (d) Defects in centromere cohesion disrupt the back-toback geometric constraints on sister kinetochores which increases the formation rate of improper kinetochore-microtubule attachments. 